\title{
A Modified Approach to Fitting Relative Importance Networks
}

\author{
Michael Brusco* \\ Florida State University \\ mbrusco@fsu.edu \\ Douglas Steinley \\ University of Missouri \\ steinleyd@missouri.edu
}

\begin{abstract}
Relative importance networks have played an important role in network psychometrics because they enable researchers to examine directional relationships among items. Most researchers have estimated the network edge weights using a well-established measure of general dominance for multiple regression and we recommend continuation of this practice. However, we recommend a modified approach that uses best-subsets regression as a preceding step to select an appropriate subset of predictors for each item. The benefits of this modified approach include: (1) a principled approach to edge selection for the relative importance network that reduces overfitting, (2) greater explained variation for scale items in comparison to the cutting of complete graphs (3) a signed network is possible if desired, and (4) potential generalization for logistic regression in the case of binary measurements. We describe and demonstrate the proposed approach and discuss its strengths and limitations.
\end{abstract}

\section{Keywords}

network psychometrics, relative importance networks, best-subsets regression; dominance analysis

*Correspondence regarding this preprint should be addressed to Michael J. Brusco, Department of Business Analytics, Information Systems, and Supply Chain, Florida State University, 821 Academic Way, Tallahassee, FL 32306, USA. 
There are several different types of network models that can be applied to psychological data (Forbes et al., 2017, 2019; Fried et al., 2017; McNally, 2016): (1) association networks (Epskamp et al., 2012), which are commonly computed as zero-order correlations between items, (2) concentration networks (van Borkulo et al., 2014), which are based on commonly-based on partial correlations, (3) directed acyclic graphs (Scutari, 2010), which are estimated using Bayesian methods and produce edges that indicate that the presence of one item is likely to suggest the presence of another item, and (4) relative importance networks (Grömping, 2006), which are based on the proportion of variance (e.g., $R^{2}$ ) one item explains in another item after controlling for all other items. The first two classes of models naturally produce graphs with undirected edges, whereas the latter two generate graphs with directed edges. Of particular interest here are relative importance networks.

Relative importance networks have been estimated using the relaimpo R software package developed by Grömping (2006), using the lmg metric (Bernstein et al., 2017; Bryant et al., 2017; Heeren \& McNally, 2016; Hoorelbeke et al., 2016; McNally et al., 2015; Robinaugh et al., 2014). The lmg metric was originally proposed by Lindeman et al. (1980), and is equivalent to the measure of general dominance described by Budescu (1993). Dominance analysis is recognized as one of the most prominent approaches to the measurement of predictor importance in regression analysis (Azen \& Budescu, 2003; Grömping, 2015; Nimon \& Oswald, 2013).

Computation of the $1 \mathrm{mg}$ measure is accomplished using all-possible-subsets regression analysis. Each of the items $j(1 \leq j \leq p)$ is taken in turn as the dependent variable and an allpossible subsets regression analysis using the other $p$-1 items as predictors is used to obtain the incoming directed edge weights for item $j$ based on the lmg measure. For each item, the number of regressions required is $2^{(p-1)}-1$. Such an approach is computationally feasible for many 
psychopathology studies where $p \leq 25$. All-possible-subsets regression might not be feasible for larger $p$. For example, in the case of $p=40$, for each item as the dependent variable, more than 500 billion regressions would be required.

In addition to scalability issues when $p$ is large, there are three other issues to consider regarding the estimation of relative importance networks using relaimpo: (1) a directed network is obtained, (2) because the edge weights are $R^{2}$ shares, which are explained variance measures, no negative edge weights are established, and (3) a complete graph is obtained because there are typically non-zero $R^{2}$ shares for each predictor of each item, The first of these issues is not a problem. In fact, we believe that it is a desirable property because it can help to assess directionality in explained variance and also help to evaluate the tenability of using a directed network. The second issue might not be a daunting problem because, in some instances, researchers focus on the absolute values of the edge-weights in their follow-up analysis. Nevertheless, in circumstances where a signed relative importance network is appropriate, a plausible approach to produce one would be desirable.

The third issue is the most problematic. If the number of items is not too large, then displaying the complete graph obtained by an all-possible-subsets regression analysis might not be too distracting. However, for larger networks, it is likely that most researchers would prefer a sparse graph for interpretation rather than a complete graph. Moreover, the complete graph often represents an egregious overfitting of the network, with many $R^{2}$ shares near zero. As recently noted in the network psychometrics literature, it is desirable " $(\mathrm{t}) \mathrm{o}$ control for spurious connections that may result from sampling error and to estimate sparser and more interpretable network models" (van den Berg et al., 2020, p. 544). One approach for creating a sparse graph is to set an arbitrary threshold for the $R^{2}$ shares. For example, in their study of bereavement 
disorder, Robinaugh et al. (2014, p. 516) used a cutoff threshold of .05 to produce their relative importance network. In their study of remitted depression, Hoorelbeke et al. (2016, p. 100) adopted a different approach for inducing sparsity in the relative importance network. Specifically, these authors only considered the edges that were selected by the concentration network when producing their relative importance graph.

We endorse the continued use of the general dominance (lmg) relative importance measure that has been used extensively in the literature. At the same time, we believe that there is an opportunity to significantly improve the process for using this measure to establish relative importance networks. This opportunity is based on the distinction between model selection and the measure of predictor importance as articulated by Budescu (1993) and Azen and Budescu (2003). In accordance with Budescu's (1993) discussion, we propose that a model selection process should precede the use of an algorithm for general dominance analysis. Different approaches for model selection have been used in the network psychometrics literature, most notably $l_{1}$-regularization methods. However, in light of recent concerns raised about the use of $l_{1-}$ regularization (Williams \& Rast, 2020; Williams et al., 2019), we propose using a nonregularized model selection process based on best-subsets regression (Brusco et al., 2009; Furnival \& Wilson, 1974; Miller, 2002). Accordingly, our proposed methodology also draws from the recent work of Williams et al. (2019) on nonregularized approaches to network estimation. Best subsets regression is a cleaner, more definitive method for model selection than regularized regression. Also, because best subsets regression and dominance analysis rely, respectively, on implicit and explicit evaluation of all-possible subsets, our method also bears a direct relationship to the use of combinatorial data analysis methods in the analysis of psychopathological data (see, for example, Loeffelman et al., 2020). 
A preceding step that uses best-subsets regression to select a subset of items as predictors for each item (when that item is the dependent variable) has several desirable properties. First, and most importantly, the subset selection process can mitigate the overfitting problem by producing a graph that is not complete and, in some cases, is rather sparse. Second, the directional sign of each predictor can be stored and used to produce a signed network if desired. Three other desirable features of the proposed approach are: (1) scalability of all-possible-subsets for computing the lmg metric is often improved because only a subset of predictors are chosen for each item, (2) a cleaner assessment of asymmetry can be made without having to compare the relative magnitude of $R^{2}$ shares (i.e., using our procedure, and edge might be selected for $\mathrm{A} \rightarrow \mathrm{B}$; but no such edge might be selected for $\mathrm{B} \rightarrow \mathrm{A}$ ), and (3) the approach is generalizable to logistic regression when the data measurements are binary.

In the next section, we describe the proposed methodology; a modification of the traditional approach to fitting relative importance networks that incorporates a preliminary step using best subsets regression. This is followed by an application of the method to the estimation of a relative importance networks for two datasets from the recent psychological literature: (i) measurements for $p=20$ items corresponding to the Toronto Alexithymia scale (Bagby et al., 1994; Briganti \& Linkowski, 2019), and (ii) measurements for $p=28$ empathy items associated with the interpersonal reactivity index (Briganti et al., 2018; Davis, 1980). The paper concludes with a brief summary and a discussion of limitations and extensions.

\section{Methodology}

\section{A general overview}

To present our methodology, we define $P=\{1, \ldots, p\}$ as a set of $p$ items under study. The data consist of real-valued measurements for each of $n$ respondents on the $p$ items, and are arranged 
in an $n \times p$ matrix, $\mathbf{X}=\left[x_{i j}\right]$. We will use the notation $\mathbf{X}(P \backslash\{q\})$ to denote the same data matrix, but excluding the column that corresponds to item $q$, and $\mathbf{x}_{q}$ will represent the vector corresponding to column $q$ in $\mathbf{X}$. The $p \times p$ matrices $\mathbf{E}=\left[e_{j k}\right]$ and $\mathbf{F}=\left[f_{j k}\right]$ will contain, respectively, the estimated edge weights and the signs (+1 or -1$)$ associated with those weights.

Step 0. Initialization. Set $q=0, \mathbf{E}=0$, and $\mathbf{F}=0$.

Step 1. Best-subsets regression analysis for the next item. Set $q=q+1$. Set $\mathbf{y}=\mathbf{x}_{q}$ and run best-subsets regression using $\mathbf{X}(P \backslash\{q\})$ as the set of candidate predictors. Select the bestsubset, $S$, based on Mallows' $C_{p}$ (Mallows, 1973), Akaike's Information Criterion (AIC: Akaike, 1973), or the Bayesian Information Criterion (BIC: Schwartz, 1978) as desired. ${ }^{1}$ Set $f_{j q}=1$ if $j \in$ $S$ and its coefficient is positive, $f_{j q}=-1$ if $j \in S$ and its coefficient is negative, and $f_{j q}=0$ if $j \in S$.

Step 2. General dominance analysis to obtain edge weights. Using $\mathbf{y}$ as the dependent variable and only those items in $S$ as predictors, run all-possible subsets regression to obtain the $R^{2}$ shares associated with a general dominance analysis. Set $e_{j q}$ equal to its corresponding $R^{2}$ share for all $j \in S$.

Step 3. If $q<p$, then go to Step 1; otherwise, if a signed network is desired the set $\mathbf{E}=\mathbf{E}$ - F, where ' $\bullet$ ' denotes the element-wise produce of the matrices, and STOP. [Note: computing the element-wise product of $\mathbf{E}$ and $\mathbf{F}$ is optional; in our analysis herein, we adopt the more common practice of ignoring the signs and working directly with the $R^{2}$ shares in $\left.\mathbf{E}\right]$.

Step 0 initializes the item index $(q=0)$ and the edge-weight $(\mathbf{E}=\mathbf{0})$ and edge-sign $(\mathbf{F}=$ 0) matrices. The item index is advanced $(q=q+1)$ in Step 1 and the column of the data matrix associated with that item is installed as the dependent variable $\left(\mathbf{y}=\mathbf{x}_{q}\right)$. Best-subsets regression analysis is then applied using all other items $(P \backslash\{q\})$ as possible predictors. The signs of the

\footnotetext{
${ }^{1}$ Selection based on the minimum value of Mallows' $C_{p}$ is equivalent to selection based on the AIC.
} 
coefficients of the selected variables $(S)$ are stored in column $q$ of matrix $\mathbf{F}$. A general dominance analysis is completed in Step 2 using $\mathbf{y}$ as the dependent variable and only the items in $S$ as the predictors. The $R^{2}$ shares obtained by the dominance analysis are stored in column $q$ of matrix E. If $q<p$ in Step 3, then there are more items remaining to serve as the dependent variable and processing returns to Step 1. If $q=p$, then estimation of $\mathbf{E}$ and $\mathbf{F}$ is complete. In many applications, there are few (if any) negative edge weights and the focus is often restricted to the absolute-values of the edge weights. For such applications, the edge-sign matrix $\mathbf{F}$ can be discarded. However, if a signed network is desired, then the element-wise product of $\mathbf{E}$ and $\mathbf{F}$ can be obtained $(\mathbf{E}=\mathbf{E} \bullet \mathbf{F})$.

We implemented the above procedure in MATLAB (MATLAB, 2020). The main program is the m-file modified_relative_importance.m, which requires only the network data as input. ${ }^{2}$ The two key components of the proposed methodology are the best subsets algorithm in Step 1 and the dominance algorithm in Step 2. The implementations of these two algorithms are discussed, respectively, in the next two subsections.

\section{Best-subsets algorithm}

The problem of selecting a subset of predictors for a regression analysis has an extensive history in the literature. Stepwise regression procedures (Efroymson, 1960) successively add and/or remove predictors until some termination criterion is achieved. The suboptimality of stepwise methods is well-known (Miller, 2002) and, accordingly, best-subsets regression approaches are preferable. Some best-subsets approaches explicitly evaluate all possible subsets of predictors (Garside, 1971; LaMotte \& Hocking, 1970; Smith, 1991), whereas others use branch-and-bound

\footnotetext{
${ }^{2}$ We also make available an $\mathrm{m}$-file relative_importance.m, which fits the relative importance using all predictors for each item and is, therefore, consistent with Grömping's (2006) relaimpo R software package that has been used in the psychological literature.
} 
programming (Brusco et al., 2009; Furnival \& Wilson, 1974) to implicitly evaluate all subsets. Using the branch-and-bound approach, many subsets are pruned (or eliminated) from the search process because it is established that they cannot possibly lead to the best subset. In our method, the number of possible predictors for each regression analysis in Step 1, the number of possible subsets is $2^{p-1}$ (including the null model with no predictors). Explicit enumeration of all-possiblesubsets would be possible for many applications in the psychopathology literature; however, the computational burden could be quite substantial when $p>25$. Therefore, we use a branch-andbound method described by Brusco et al. (2009), which is more efficient and typically feasible for up to $p \leq 40$.

The branch-and-bound algorithm will find the subset of $v$ predictors that minimizes the error/residual sum-of-squares (or, equivalently, maximizes $R^{2}$ ) for each number of predictors on the interval $1 \leq v \leq p$. It is in this sense that we have identified the "best" subset of predictors for each value of $v$. However, because $R^{2}$ is monotonically non-decreasing as a function of $v$, the problem of selecting the best value of $v$ remains. There are a variety of indices that can be used to select the appropriate value of $v$, such a Mallows' $C_{p}$, AIC, or BIC. If the minimum value of Mallows' $C_{p}$ is used as the criterion, then this measure leads to the same selection as the AIC.

The branch-and-bound method is implemented in the MATLAB m-file batch_bestsub.m, which runs the best-subsets analysis for all subset sizes $1 \leq v \leq p$. For each subset size, the identification of the best subset is obtained using the bestsubh.m subroutine that is called by batch_bestsub.m. Moreover, the bestsubh.m subroutine calls the subroutine sweep.m for efficient computation of $R^{2}$ for the regression models (Goodnight, 1979). The selection of the best value of $v$ and the corresponding selected subset $S$ in Step 2 of 
the algorithm is controlled by the modified_relative_importance.m main program and the choice can be made based on either Mallows' $C_{p}$ or BIC.

\section{Dominance analysis algorithm}

Dominance analysis is closely related to best-subsets regression in the sense that it requires the explicit evaluation of all possible subsets of predictors. In Step 3 of our method, dominance analysis is based on the evaluation of all $2^{v}$ subsets associated with $S$. The fact that $v$ is often significantly smaller than $p$-1 helps to ease the computational burden of evaluating all possible subsets. Some authors have framed the implementation of dominance analysis and the computation of the lmg measure in terms of the evaluation of all $v$ ! sequences (or permutations) of the predictors (Bulteel et al., 2016; Fried \& Nesse, 2014; Grömping, 2006). Although such descriptions are correct, it is important to recognize that enumeration of all sequences is not necessary because the $R^{2}$ share contribution when adding a predictor, $j$, to a subset, $S^{\prime}$, does not depend on the order in which the predictors in $S^{\prime}$ were entered. The focus on subsets rather than permutations is crucial because the evaluation of $2^{v}$ regression models is computationally feasible for $v \leq 25$ (possibly $v \leq 30$ depending on the hardware/software platform), whereas the evaluation of $v$ ! regressions would only be feasible for roughly $v \leq 15 .^{3}$

Budescu (1993) described three different types of dominance. A predictor $j$ exhibits complete dominance over another predictor $j^{\prime}$ if, for any subset $T \subset S: j \notin T \wedge j^{\prime} \notin T$, adding $j$ to $T$ will improve $R^{2}$ more than adding $j^{\prime}$ to $T$. This is the strongest type of dominance because it means that, for any subset of predictors that does not include $j$ and $j^{\prime}$, adding $j$ will always be

\footnotetext{
${ }^{3}$ Technically, the algorithm does not recompute separate regressions from scratch. Instead, the $R^{2}$ computations for the different subsets of predictors are made by applying the sweep algorithm to the correlation matrix.
} 
result in a greater increase in $R^{2}$. The second type of dominance, known as conditional dominance, is based on the examination of the average improvement in $R^{2}$ for each possible subset size. If the average improvement in $R^{2}$ is greater for $j$ than $j^{\prime}$ for all subset sizes $(1, \ldots, v)$, then $j$ has conditional dominance over $j^{\prime}$. The third type of dominance pertains to the $1 \mathrm{mg}$ measure (Lindeman et al., 1980) and is referred to as general dominance. This measure can be computed as the average (across all subset sizes) of the subset-size averages computed for conditional dominance. Of the three dominance measures, only general dominance directly provides a definitive ranking of the predictors with respect to importance. Brusco et al. (2019) have recently proposed a dynamic programming approach for ranking the predictors based on the stronger conditions of complete and conditional dominance.

The dominance analysis algorithm is implemented in the MATLAB m-file dominance.m, which runs dominance analysis via complete enumeration for all subset sizes $1 \leq$ $i \leq v$. The dominance. $\mathrm{m}$ algorithm is called immediately after running the batch_bestsub.m subroutine for each item $(q)$ and is applied using the $v$ variables selected for that item. The $R^{2}$ shares obtained from the dominance analysis are used to populate column $q$ of $\mathbf{E}$ as described in Step 2 of the algorithm above. Column $q$ of $\mathbf{F}$ is populated with the sign of the predictors when the $v$ variables are used to predict item $q$.

\section{Interpretation of output}

The primary outputs of the modified_relative_importance.m program are the matrix of

edge weights, E, and the matrix of signs, F. We have also incorporated a MATLAB m-script that calculates several summary statistics for $\mathbf{E}$ : (1) dee - the total number of directed edges estimated, (2) mie - the minimum number of incoming directed edges across all items, (3) mxethe maximum number of directed edges across all items, (4) uee - the total number of 
unreciprocated edges estimated, (5) mxr - the maximum $R^{2}$ across all items (6) avr - the average $R^{2}$ across all items, (7) mir - the minumum $R^{2}$ across all items, (8) mxs - the maximum $R^{2}$ share across all edges in the estimated network (9) avs - the average $R^{2}$ share across all edges in the estimated network, and (10) mis - the minumum $R^{2}$ share across all edges in the estimated network.

\section{Illustrative Examples}

\section{Datasets}

We applied the proposed method to two datasets from the literature. The specific items for the datasets are described in Table 1. The first dataset was published by Briganti and Linkowski (2019) and pertains to $p=20$ items for the Toronto Alexithymia scale (Bagby et al, 1994). Measurements were obtained for each of $n=1925$ respondents on each of the items on a scale of 1 "I completely disagree" to "I completely agree". The second dataset was published by Briganti et al. (2018) and corresponds to $p=28$ empathy items associated with the interpersonal reactivity index (Davis, 1980). Measurements for $n=1973$ respondents were obtained for each of the items on a scale of 0 (“doesn't describe me very well”) to 4 (“describes me very well”). The original analyses of both of these datasets were performed using concentration networks. More specifically, Gaussian graphical models were estimated using a regularized partial correlation method (Epskamp \& Fried, 2018). To the best of our knowledge, relative importance networks have not previously been fit to these datasets.

For each dataset, we began by fitting the standard approach used in the literature, which is to fit the full relative importance network (a complete graph) using relative_importance.m. We verified (for the alexithymia data) that this program produces the same estimates as relaimpo using the lmg metric and, hereafter, we refer to this method as RI. Next, we applied 
our proposed method modified_relative_importance.m twice to each dataset: (i) once

using Mallows's $C_{p}$ for choosing the subset sizes (MRI-CP), and (ii) once using BIC for choosing the subset sizes (MRI-BIC).

Table 1. Summary Description of Items for the Empirical Datasets

\begin{tabular}{|c|c|c|}
\hline Item & Alexithymia items (Briganti \& Linkowski, 2019) & Empathy items (Briganti et al. 2018) \\
\hline 1 & confused about emotion I'm feeling & daydream and fantasize with regularity (fantasy) \\
\hline 2 & difficult to find right words for my feelings & tender feelings for less fortunate (empathetic concern) \\
\hline 3 & physical sensations doctors don't understand & tough to see others point of view (perspective taking) \\
\hline 4 & able to describe my feelings easily & don't feel sorry for others (empathetic concern) \\
\hline 5 & prefer to analyze problems rather than just describe them & involved with novel character's feelings (fantasy) \\
\hline 6 & don't know whether I'm sad, frightened, or angry & apprehensive in emergencies (personal distress) \\
\hline 7 & often puzzled by sensations in my body & objective watching movie or play (fantasy) \\
\hline 8 & prefer to just let things happen rather than find out why & look at everybody's side (perspective taking) \\
\hline 9 & have feelings I can't quite identify & protective of one taken advantage of (empathetic concern) \\
\hline 10 & being in touch with emotions is essential & helpless in emotional situations (personal distress) \\
\hline 11 & find it hard to describe my feelings more & imagine from other's perspective (perspective taking) \\
\hline 12 & people tell me to describe my feelings more & rare to be involved with movie or book (fantasy) \\
\hline 13 & don't know what's going on inside me & remain calm when someone hurt (personal distress) \\
\hline 14 & often don't know why I am angry & other's misfortunes don't disturb me (empathetic concern) \\
\hline 15 & prefer talking to others about activities over feelings & when right, don't listen to others (perspective taking) \\
\hline 16 & prefer to watch 'light' shows rather than dramas & feel like one of play/movie characters (fantasy) \\
\hline 17 & difficult to reveal innermost feelings to close friends & tense emotional situations scare me (personal distress) \\
\hline 18 & can feel close to someone even in moments of silence & no pity for unfairly treated (empathetic concern) \\
\hline 19 & find examining my feelings useful for solving my problems & effective dealing with emergencies (personal distress) \\
\hline 20 & looking for meaning in movies/plays distracts enjoyment & touched by things I see happen (fantasy) \\
\hline 21 & & look at both sides to every question (perspective taking) \\
\hline 22 & & a pretty soft-hearted person (empathetic concern) \\
\hline 23 & & put myself in leading character of a movie (fantasy) \\
\hline 24 & & tend to lose control during emergencies (personal distress) \\
\hline 25 & & "put myself I his shoes" when upset (perspective taking) \\
\hline 26 & & feel as if events in story/novel happen to me (fantasy) \\
\hline 27 & & go to pieces in emergency (personal distress) \\
\hline 28 & & imagine how I'd feel in their place (perspective taking) \\
\hline
\end{tabular}

\section{Results}

Comparison of RI, MRI-CP, and MRI-BIC. Table 2 presents a summary of the results obtained using the RI, MRI-CP, and MRI-BC methods for both the alexithymia and empathy datasets. The 
summary includes the measures described in the previous section, as well as computation time. ${ }^{4}$ The RI method estimated complete graphs for both datasets, consisting of dee $=380$ and dee $=$ 756 directed edges, respectively. In light of the fact that the graph is complete, there are no unreciprocated edges and the number of items selected for prediction is always $p-1$. For both networks, the MRI-CP approach obtained networks with approximately $53 \%$ of the number of edges in the complete graph, whereas the MRI-BIC implementation obtained networks with approximately $29 \%$ of the number of edges in the complete graph. Despite this substantial reduction in the density of the estimated network, the results in Table 2 show that the sacrifice in explained variation is quite small. For the alexithymia dataset, the average $R^{2}$ value for RI (using all items as predictors) is .2454 . The average dips only slightly to .2432 for MRI-CP using roughly half as many predictors, and to .2352 for MRI-BIC using just under $30 \%$ of the predictors. Likewise, for the empathy dataset, the average $R^{2}$ value for RI is .2739 . The average falls slightly to .2709 for MRI-CP and to .2609 for MRP-BIC.

The $R^{2}$ results in Table 2 suggest that the complete graph associated with RI is indicative of overfitting, as nearly the same level of variation can be obtained with far fewer edges in the network. Forcing more edges into the network also results in a significant reduction of the average $R^{2}$ values that represent the edge weights. The average $R^{2}$ shares (avs) for the alexithymia and empathy networks are .0129 and .0101, respectively. Contrastingly, these averages more than triple to .0424 and .0332 , respectively, when using the MRI-BIC approach. Comparison to Subgraphs of RI. As noted in the Introduction, different approaches have been used to produce a sparser graph from the complete graph obtained by relaimpo. One approach is to delete all edges that do not meet some threshold. For direct comparison to the MRI-CP and

\footnotetext{
${ }^{4}$ All methods were implemented on a desktop computer with an Intel ${ }^{\circledR}$ Core ${ }^{\text {TM }}$ i7-1051CPU @ $1.8 \mathrm{GHz}$ with 16 GB of RAM.
} 
MRI-BIC results, we deleted edges in the complete graphs obtained by RI, beginning with the smallest $R^{2}$ share value and continuing in ascending order until the number of directed edges was equal to the number in the MRI-CP or MRI-BIC solution as desired. We refer to these as subgraphs of RI (i.e., graphs obtained by removing some of the edges from the complete graph produced by RI). More specifically, we define RI-cp as the subgraph of RI that has the same number of edges as MRI-CP, and RI-bic as the subgraph of RI that has the same number of edges as MRI-BIC.

Table 2. Computational Results for the Empirical Datasets.

\begin{tabular}{|c|c|c|c|c|c|c|}
\hline & \multicolumn{3}{|c|}{ Alexithymia data $(p=20)$} & \multicolumn{3}{|c|}{ Empathy data $(p=28)$} \\
\hline & RI & MRI-CP & MRI-BIC & RI & MRI-CP & MRI-BIC \\
\hline Num. directed edges (dee) & 380 & 203 & 111 & 756 & 406 & 220 \\
\hline Min. number of edges per symptom (mie) & 19 & 7 & 3 & 27 & 10 & 4 \\
\hline Max. number of edges per symptom (mxe) & 19 & 13 & 9 & 27 & 20 & 13 \\
\hline Num. unreciprocated edges (uee) & 0 & 11 & 13 & 0 & 30 & 24 \\
\hline Max. $R^{2}$ across symptoms (mxr) & .5414 & .5397 & .5346 & .4602 & .4579 & .4518 \\
\hline Avg. $R^{2}$ across symptoms (avr) & .2454 & .2432 & .2352 & .2739 & .2709 & .2609 \\
\hline Min. $R^{2}$ across symptoms (mir) & .0817 & .0793 & .0684 & .1209 & .1182 & .0947 \\
\hline Max. $R^{2}$ share (mxs) & .2165 & .2440 & .2649 & .1910 & .2014 & .2133 \\
\hline Avg. $R^{2}$ share (avs) & .0129 & .0240 & .0424 & .0101 & .0187 & .0332 \\
\hline Min. $R^{2}$ share (mis) & .0001 & .0006 & .0020 & .0001 & .0005 & .0018 \\
\hline Computation time in seconds & 49.08 & 5.24 & 4.90 & 25453.18 & 64.46 & 52.90 \\
\hline
\end{tabular}

Note - the columns labeled 'RI' contain results for the standard approach for relative importance networks using all predictors with the lmg measure. The columns labeled 'MRI-CP' and 'MRI-BIC' correspond to our modified relative importance approach using Mallows' $C_{p}$ and BIC for best-subsets variable selection, respectively.

For both the alexithymia and empathy datasets, Table 3 provides the $R^{2}$ values for each item for a fixed number of edges, as obtained by MRI directly using either $C_{p}$ or BIC for model 
selection, or corresponding to the subgraphs of RI created by eliminating its smallest edges. In addition, two 203-edge network graphs for the empathy data, which were produced using qgraph (Epskamp et al., 2012) are displayed in Figures 1 and 2. Figure 1 corresponds to the MRI-BIC solution, whereas Figure 2 is associated with RI-bic (i.e., the subgraph of RI after eliminating edges so as to have the same number as the MRI-BIC solution).

The results in Table 3 show that the proposed modified method for relative importance networks can result in a substantial improvement in explained variation relative to cutting the complete graph obtained from a relative importance analysis based on all predictors. For both the alexithymia and empathy networks, for all items, and regardless of whether the number of edges was based on $C_{p}$ or BIC, MRI yielded a better $R^{2}$ value than the subgraph of RI. For the alexithymia dataset, the $R^{2}$ values for RI averaged (across all items) 3.9\% and 14.5\% lower than the corresponding $R^{2}$ values for MRI using $C_{p}$ and BIC, respectively. Comparable averages of $4.6 \%$ and $14.2 \%$ were measured for the empathy dataset. Moreover, Table 3 reveals that the sacrifice in explained variation associated with the RI subgraph was unevenly distributed across the items. For example, for the alexithymia dataset, the minimum sacrifice across the items for the 111-edge network associated was $4.0 \%$ (for item 9) and the maximum was $39.6 \%$ (for item 18). Even in the case of item 9 , where the $R^{2}$ sacrifice was minimal, the rank-order of predictor importance (i.e., $R^{2}$-shares) for item 9 was 13-1-2-11-7-6-8 for the MRI solution, but 13-1-2-7-411-6-14-2-8 for the RI solution. Whereas item 11 ranks ahead of item 7 in the MRI solution, it ranks behind item 7 (and item 4) in the RI solution. Item 11's position in the rank order if the RI solution Thus, the overfitting associated with RI can not only lead to reduced explanatory power, but also errant conclusions regarding the relative importance of predictors. 
Table 3. A Comparison of $R^{2}$ Values for Each Item when the Relative Importance Network is Cut to Have the Same Number of Edges as the Best-Subsets Solution.

\begin{tabular}{|c|c|c|c|c|c|c|c|c|c|c|c|c|}
\hline \multirow[b]{3}{*}{ Item } & \multicolumn{6}{|c|}{ Alexithymia data $(p=20)$} & \multicolumn{6}{|c|}{ Empathy data $(p=28)$} \\
\hline & \multicolumn{3}{|c|}{203 edges (from Cp) } & \multicolumn{3}{|c|}{111 edges (from BIC) } & \multicolumn{3}{|c|}{406 edges (from Cp) } & \multicolumn{3}{|c|}{220 edges (from BIC) } \\
\hline & MRI-Cp & $\mathrm{RI}-\mathrm{Cp}$ & $\% \Delta$ & MRI-bic & RI-bic & $\% \Delta$ & MRI-Cp & $\mathrm{RI}-\mathrm{Cp}$ & $\% \Delta$ & MRI-bic & RI-bic & $\% \Delta$ \\
\hline 1 & .3955 & .3884 & 1.8 & .3916 & .3628 & 7.4 & .1182 & .1091 & 19.8 & .0947 & .0718 & 24.2 \\
\hline 2 & .5397 & .5341 & 1.0 & .5346 & .5096 & 4.7 & .2876 & .2783 & 2.0 & .2819 & .2314 & 17.9 \\
\hline 3 & .1636 & .1553 & 5.1 & .1548 & .1162 & 25.0 & .1864 & .1759 & 5.6 & .1759 & .1466 & 16.6 \\
\hline 4 & .4586 & .4506 & 1.7 & .4549 & .4181 & 8.1 & .3097 & .2972 & 3.1 & .3000 & .2743 & 8.6 \\
\hline 5 & .1361 & .1283 & 5.7 & .1283 & .1163 & 9.4 & .3443 & .3347 & 2.6 & .3355 & .3087 & 8.0 \\
\hline 6 & .2022 & .2016 & .3 & .1964 & .1738 & 11.6 & .2907 & .2776 & 2.1 & .2847 & .2573 & 9.6 \\
\hline 7 & .2212 & .2114 & 4.5 & .2108 & 1900 & 9.9 & .1963 & .1833 & 7.2 & .1823 & .1436 & 21.3 \\
\hline 8 & .1465 & .1366 & 6.8 & .1359 & .1214 & 10.7 & .2439 & .2316 & 3.7 & .2348 & .2020 & 13.9 \\
\hline 9 & .3843 & .3786 & 1.5 & .3805 & .3651 & 4.0 & .2803 & .2691 & 4.1 & .2688 & .2467 & 8.2 \\
\hline 10 & .1231 & .1124 & 8.7 & .1091 & .0750 & 31.3 & .3388 & .3310 & 0.4 & .3376 & .2890 & 14.4 \\
\hline 11 & .2408 & .2343 & 2.7 & .2294 & .2067 & 9.9 & .2493 & .2349 & 5.3 & .2362 & .2062 & 12.7 \\
\hline 12 & .2505 & .2444 & 2.4 & .2422 & .2149 & 11.3 & .2505 & .2357 & 4.4 & .2394 & .2254 & 5.9 \\
\hline 13 & .4152 & .4086 & 1.6 & .4096 & .3911 & 4.5 & .2515 & .2413 & 4.6 & .2399 & .2084 & 13.1 \\
\hline 14 & .2255 & .2207 & 2.1 & .2198 & .1791 & 18.5 & .3865 & .3725 & 1.2 & .3820 & .3416 & 10.6 \\
\hline 15 & .1870 & .1806 & 3.4 & .1755 & .1368 & 22.0 & .1309 & .1150 & 13.8 & .1129 & .0776 & 31.3 \\
\hline 16 & .1425 & .1371 & 3.8 & .1343 & .1188 & 11.6 & .3880 & .3745 & 1.2 & .3832 & .3571 & 6.8 \\
\hline 17 & .2612 & .2547 & 2.5 & .2542 & .2220 & 12.7 & .3057 & .2981 & 3.7 & .2944 & .2497 & 15.2 \\
\hline 18 & .0793 & .0700 & 11.8 & .0684 & .0413 & 39.6 & .1852 & .1706 & 8.0 & .1704 & .1480 & 13.2 \\
\hline 19 & .1696 & .1586 & 6.5 & .1596 & .1434 & 10.2 & .2616 & .2544 & 1.4 & .2579 & .2226 & 13.7 \\
\hline 20 & .1225 & .1175 & 4.1 & .1132 & .0818 & 27.8 & .3250 & .3153 & 3.2 & .3147 & .2696 & 14.3 \\
\hline 21 & & & & & & & .1470 & .1338 & 8.0 & .1353 & .1039 & 23.2 \\
\hline 22 & & & & & & & .2671 & .2585 & 4.4 & .2552 & .2188 & 14.3 \\
\hline 23 & & & & & & & .4579 & .4383 & 1.3 & .4518 & .4240 & 6.1 \\
\hline 24 & & & & & & & .3057 & .2888 & 2.4 & .2985 & .2676 & 10.4 \\
\hline 25 & & & & & & & .2180 & .2037 & 3.6 & .2102 & .1760 & 16.3 \\
\hline 26 & & & & & & & .3720 & .3641 & 3.0 & .3608 & .3151 & 12.7 \\
\hline 27 & & & & & & & .2358 & .2242 & 6.7 & .2199 & .1899 & 13.6 \\
\hline 28 & & & & & & & .2522 & .2479 & 2.6 & .2456 & .1915 & 22.1 \\
\hline Max & .5397 & .5341 & 11.8 & .5346 & .5096 & 39.6 & .4579 & .4383 & 19.8 & .4518 & .4240 & 31.3 \\
\hline Mean & .2432 & .2362 & 3.9 & .2352 & .2092 & 14.5 & .2709 & .2593 & 4.6 & .2609 & .2273 & 14.2 \\
\hline Min & .0793 & .0700 & .3 & .0684 & .0413 & 4.0 & .1182 & .1091 & 0.4 & .0947 & .0718 & 5.9 \\
\hline
\end{tabular}

Note - the table elements correspond to $R^{2}$ values for each item. The RI-cp/RI-bic columns correspond to preserving only cutting edges (proceeding from the smallest edge weight) from the complete graph associated with the standard relative importance approach, so as to match the number of edges selected by either the MRI-CP or MRI-BIC approaches, respectively. The ' $\% \Delta$ ' column is the percentage difference between RI and MRI. 


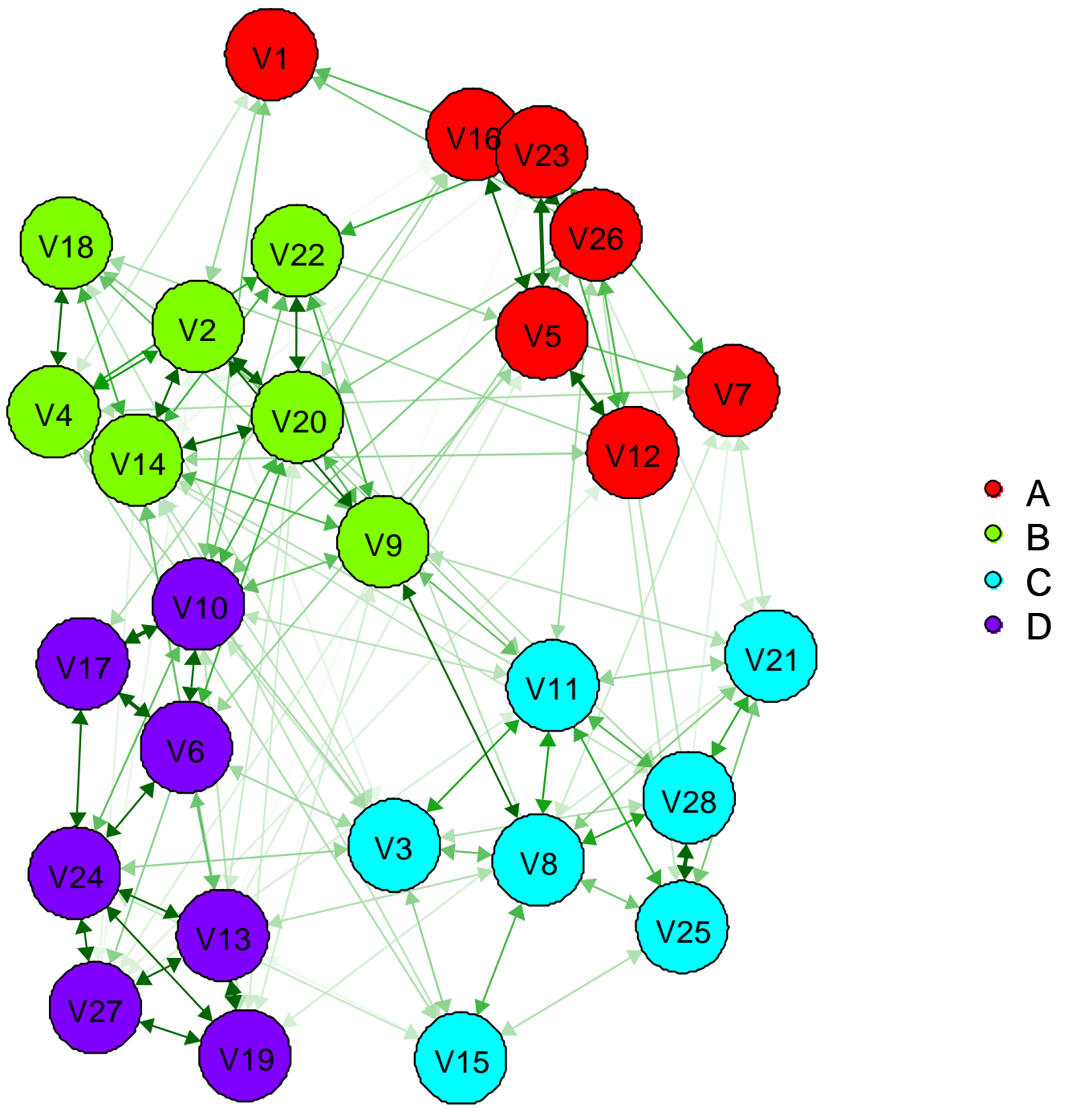

Figure 1. This is the directed graph for the empathy network obtained from the modified relative importance method using BIC with best-subsets regression (MRI-BIC). The vertex colors pertain to Davis' (1980) four subscales: $\mathrm{A}=$ fantasy, $\mathrm{B}=$ empathetic concern, $\mathrm{C}=$ perspective taking, and $\mathrm{D}=$ personal distress. 


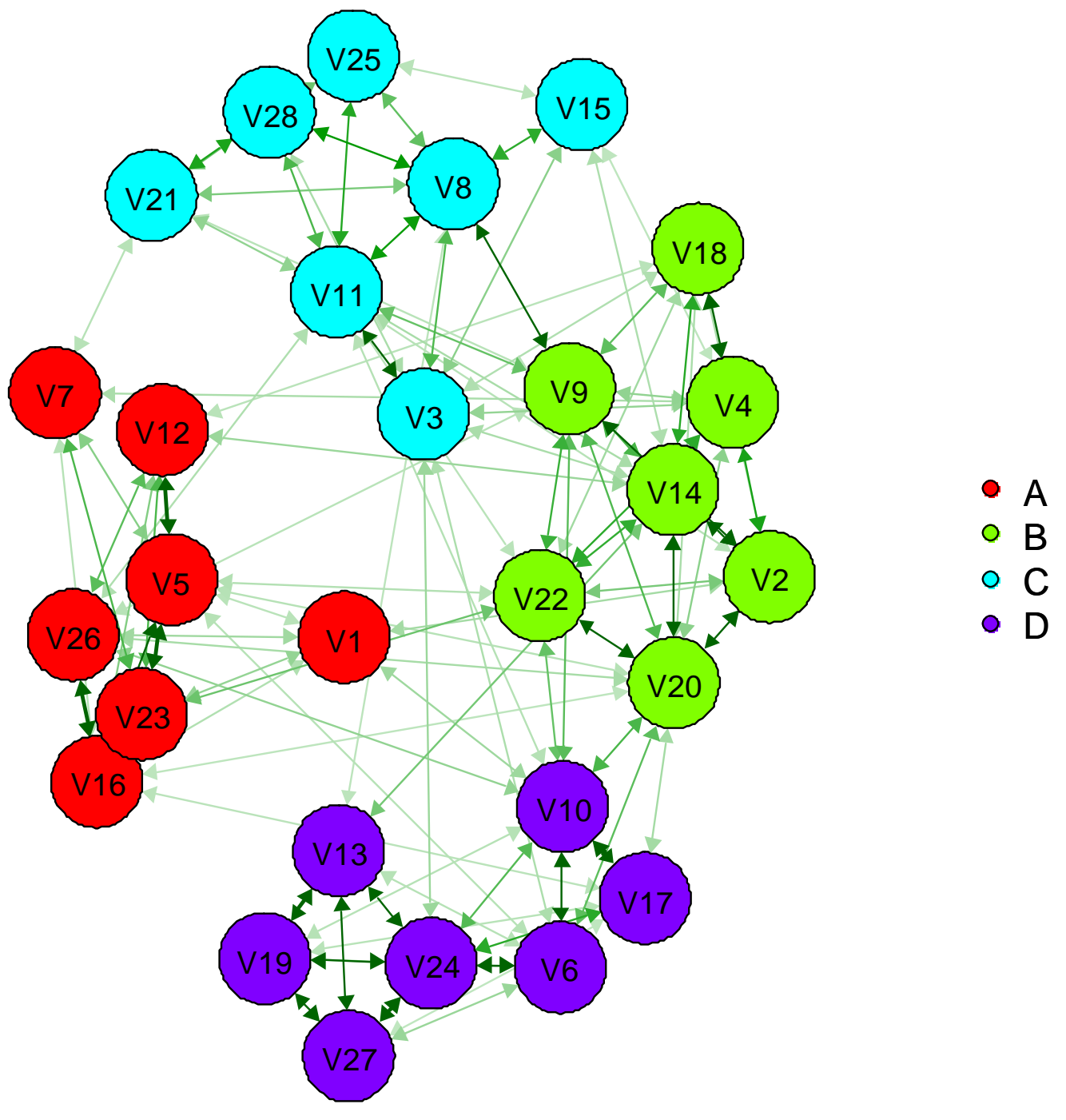

Figure 2. This is the directed graph, RI-bic, for the empathy network obtained by cutting edges (beginning with the smallest and continuing in ascending order) of the complete graph for full RI network so as to match the number of edges (220) in the MRI-BC graph in Figure 1. The vertex colors pertain to Davis' (1980) four subscales: $\mathrm{A}=$ fantasy, $\mathrm{B}=$ empathic concern, $\mathrm{C}=$ perspective taking, and $\mathrm{D}=$ personal distress.

To further examine the potential interpretative implications for using MRI vs. RI, consider item 1 in Figures 1 and 2. In the MRI-BIC graph (Figure 1), the incoming edge set for item 1 is $\{2,4,10,23,26\}$, but the incoming edge set is properly restricted to $\{2,4,10\}$ because item 1 is 
a comparatively weak predictor for items 23 and 26. For the incoming and outgoing edge set for the RI-bic subgraph (Figure 2) are both $\{2,5,10,16,23,26\}$. There are a couple of important observations regarding this result. First, the MRI approach is more likely to pick up asymmetric relationships among the items because the overfitting associated with RI tends to result in a compression of the $R^{2}$ shares. The inclusion of items 23 and 26 in the incoming edge set for item 1 is not spurious, as these two items have the largest $R^{2}$ shares for item 1 of .0271 and .0220 , respectively. These are roughly double their $R^{2}$ shares in the RI-bic solution. This leads to second observation, which is that RI subgraphs have a propensity to suppress the measure of strength of the relationships between items. In other words, not only is the total explained variation $\left(R^{2}=\right.$ $.0718)$ for item 1 in the RI-bic subgraph $24.2 \%$ less than its explained variation $\left(R^{2}=.0947\right)$ in the MRI-BIC graph, but the $R^{2}$ shares of the most important drivers (items 23 and 26) are underestimated because of the overfitting associated with RI.

$\boldsymbol{k}$-fold cross-validation. To better assess the predictive performance of the different relative importance networks for the alexithymia and empathy datasets, we conducted a $k$-fold cross validation (Constantini \& Epskamp, 2017; Fried et al., 2018). For each dataset, the $n$ respondents were randomly divided into $k=10$ folds of approximately equal size. ${ }^{5}$ Each fold was used one time as the holdout sample, with the other nine folds comprising the training sample. The performance measures of interest were the holdout-sample $R^{2}$ values for each of the $p$ items using the subsets of predictors obtained from each of the five methods described above: (i) RI all $p-1$ predictors from the standard approach to relative importance, (ii) MRI-CP - using the best-subset predictors selected based on Mallows's $C_{p}$, (iii) MRI-BIC - using the best-subset predictors selected based on BIC, (iv) RI-cp - using the predictors from the subgraph of RI that

\footnotetext{
${ }^{5}$ For example, for the empathy dataset, the $n=1973$ respondents were randomly divided into $k=10$ folds consisting of either 197 or 198 respondents.
} 
has the same number of edges as MRI-CP, and (v) RI-bic - using the predictors from the subgraph of RI that has the same number of edges as MRI-BIC.

Based on the average $R^{2}$ for the holdout sample across the $k=10$ folds, MRI-CP was the clear winner in terms of predictive performance for both datasets. For the alexithymia dataset, MRI-CP yielded a larger average $R^{2}$ than the other four models for 18 of the 20 items. Across the 20 items, the average $R^{2}$ of MRI-CP was $2.22 \%$ and $2.42 \%$ greater than the corresponding averages of RI-cp and MRI-BIC, respectively. The predictive performances of RI and RI-bic were appreciably worse. The average $R^{2}$ of MRI-CP was $3.76 \%$ and $5.87 \%$ greater than the corresponding averages of RI and RI-bic, respectively.

Likewise, for the empathy dataset, MRI-CP yielded a larger average $R^{2}$ (across the $k=10$ folds) than the other four models for 27 of the 28 items in the dataset. Across the 28 items, the average $R^{2}$ of MRI-CP was $1.74 \%$ and $1.97 \%$ greater than the corresponding averages of RI-cp and MRI-BIC, respectively. Once again, the RI and RI-bic models yielded appreciably worse predictive performance: The average $R^{2}$ of MRI-CP was $4.20 \%$ and $4.61 \%$ greater than the corresponding averages of RI and RI-bic, respectively.

Computation time. Finally, the computation time results in Table 2 should not be overlooked. It is important to recognize that both the alexithymia and empathy networks are larger than most of those tackled in the literature using Grömping's (2006) rela impo R package (Hoorelbeke et al., 2016; McNally et al., 2015; Robinaugh et al., 2014) and, accordingly, the computational demand is greater. For the 20 -item alexithymia dataset, there are $2^{19}=524,288 R^{2}$ computations that must be made for each of the 20 items serving as the dependent variable. However, for the 28 -item empathy dataset, there are $2^{27}=134,217,728 R^{2}$ computations that must be made for each of the 28 items serving as the dependent variable. Accordingly, whereas the computation time for RI is 
a modest 49.08 seconds for the alexithymia dataset, it is an enormous $25,423.18$ seconds (> 7 hours) for the empathy dataset. By contrast, the computation times for MRI-CP and MRI-BIC in Table 1 are modest for both datasets. The best-subsets variable selection process sharply reduces the number of predictors for each item and, therefore, computation of the general dominance (lmg) measure is much more efficient. This is an added benefit of our proposed method, as it might enable the fitting of relative importance networks to datasets where the number of items makes RI or relaimpo computationally infeasible.

\section{Conclusions}

\section{Summary}

General dominance analysis using the lmg metric in Grömping's (2006) $R$ software package relaimpo has been the standard approach for estimating relative importance networks in psychopathology. This is a well-accepted approach for measuring predictor importance in regression analysis and we support its continued use. Nevertheless, drawing from the work of Budescu (1993) and Azen and Budescu (2003), we believe that it is important to distinguish between model selection and predictor importance. Accordingly, we recommend using bestsubsets regression to select a subset of predictors for each item prior to estimating the edge weights for the items using general dominance analysis.

Relative to the standard approach, there are multiple advantages to our modified approach for fitting relative importance networks. First, the standard approach produces a complete graph that tends to result in overfitting, particularly for larger networks. By contrast, our modified approach selects the edges of the graph using well-established criteria for model selection in best subsets regression. Second, using a threshold to produce a sparse graph from the complete graph obtained by the standard approach is inferior to our modified approach with respect to preserving 
explained variation in the items. Third, the modified approach is better able to reveal asymmetric explanatory relationships among the items because it explicitly produces a directed graph and also has less suppression of the edge weights. Fourth, the modified approach can produce a signed graph if desired. Fifth, the modified approach is often much faster than the standard approach because of the subset-selection process and, therefore, is apt to be scalable for larger networks.

\section{Limitations and extensions}

One of the limitations of our proposed method is that it requires a model selection decision for each item in its role as the dependent variable. The BIC criterion is more conservative than Mallows' $C_{p}$ and tends to yield to a network graph that is appreciably less dense. Nevertheless, the sacrifice in explained variation for this greater parsimony tends to be rather modest. Moreover, because there are fewer edges selected but explained variation is comparable, the individual edge weights tend to be somewhat larger on average. Selection of the model based on Mallows' $C_{p}$ can be viewed as a compromise between the $\mathrm{BIC}$ and fitting the relative importance network with no model selection. For the two examples herein, the estimated networks were slightly more than $50 \%$ dense. In addition to Mallows' $C_{p}$, AIC, and BIC, there are other information criteria that could be used for model selection. As we have shown, the choice of information criterion can be based on some type of $k$-fold cross-validation (Constantini \& Epskamp, 2017; Fried et al., 2018). For the two datasets analyzed herein, $k$-fold cross-validation revealed that Mallows' $C_{p}$ resulted in better predictive performance.

We have implemented the modified relative importance method in MATLAB, which we believe is a more transparent language for users and allows for easier customization of the source code (i.e., the m-files). Nevertheless, the approach is also amenable to implementation in R. 
Grömping's (2006) rela impo R package is already familiar to many researchers in the psychological community and there are some alternative $\mathrm{R}$ packages available for best-subsets regression, such as leaps (Lumley \& Miller, 2020) and olsrr (Hebbali, 2020).

Our analyses in this paper have assumed metric variable measurements and the use of linear regression analysis for both model selection and establishment of the edge weights.

However, there are a substantial number of applications in the network psychometrics literature that focus on the analysis of binary data within the framework of the Ising model. A common estimation procedure for this model is based on regularized logistic regression (van Borkulo et al., 2014), and this approach also inherently produces an asymmetric network matrix that can be used for studying relative importance. Our proposed method can be adapted to provide a nonregularized approach for binary data. The branch-and-bound algorithm for variable selection in regression could be replaced with an all-possible-subsets approach for logistic regression (when the subset of items is, say, 20 or less) or a metaheuristic for larger subsets. The selection of the appropriate subset size could still be based on criteria such as AIC or BIC. In addition, dominance analysis has been extended to logistic regression by Azen and Traxel (2009), which might afford a viable approach for establishing the edge weights.

\section{References}

Akaike, H. (1973). Information theory and an extension of the maximum likelihood principle. In B. N. Petrov \& B. F. Csaki (Eds.) Second international symposium on information theory (pp. 267-281) Budapest: Academiai Kiado.

Azen, R., \& Budescu, D. V. (2003). The dominance analysis approach for comparing predictors in multiple regression. Psychological Methods, 8 (2), 129-148. doi:10.1037/1082989x.8.2.129

Azen, R., \& Traxel, N. (2009). Using dominance analysis to determine predictor importance in logistic regression. Journal of Educational and Behavioral Statistics, 34 (3), 319-347. doi: $10.3102 / 1076998609332754$

Bagby, R. M., Parker, J. D. A., \& Taylor, G. J. (1994). The twenty-item Toronto Alexithymia Scale-I. Item selection and cross-validation of the factor structure. Journal of Psychosomatic Research, 38 (1), 23-32. doi:10.1016/0022-3999(94)90005-1 
Bernstein, E. E., Heeren, A., \& McNally, R. J. (2017). Unpacking rumination and executive control: A network perspective. Clinical Psychological Science, 5 (5), 816-826. doi: $10.1177 / 2167702617702717$

Briganti, G., Kempenaers, C., Braun, S., Fried, E. I., \& Linkowski, P. (2018). Network analysis of empathy items from the interpersonal reactivity index in 1973 young adults. Psychiatry Research, 265, 87-92. doi: 10.1016/j.psychres.2018.03.082

Briganti, G., \& Linkowski, P. (2019). Network approach to items and domains from the Toronto Alexithymia scale. Psychological Reports. Retrieved from: https://journals.sagepub.com/doi/abs/10.1177/0033294119889586

Brusco, M. J., Cradit, J. D., \& Brudvig, S. (2019). An integrated dominance analysis and dynamic programming approach for measuring predictor importance for customer satisfaction. Communications in Statistics - Theory and Methods, 48 (21), 5290-5307. doi:10.1080/03610926.2018.1510004

Brusco, M. J., Steinley, D., \& Cradit, J. D. (2009). An exact algorithm for hierarchically wellformulated subsets in second-order polynomial regression. Technometrics, 51 (3), 306-315. doi:10.1198/tech.2009.08022

Bryant, R. A., Creamer, M., O’Donnell, M., Forbes, D., McFarlane, A. C., Silove, D., HadziPavlovic, D. (2017). Acute and chronic posttraumatic stress symptoms in the emergence of posttraumatic stress disorder a network analysis. JAMA Psychiatry, 74 (2), 135-142. doi:10.1001/jamapsychiatry.2016.3470

Budescu, D. V. (1993). Dominance analysis: A new approach to the problem of relative importance of predictors in multiple regression. Psychological Bulletin, 114 (3), 542-551.

Bulteel, K., Tuerlinckx, F., Brose, A., \& Ceulemans, E. (2016). Using raw VAR regression coefficients to build networks can be misleading. Multivariate Behavioral Research, 51 (23), 330-344, doi: 10.1080/00273171.2016.1150151

Costantini, G., \& Epskamp, S. (2017). EstimateGroupNetwork: Perform the joint graphical lasso and selects tuning parameters. R package (Version 0.1.2) [Computer software]. Retrieved from https://cran.r-project.org/web/packages/EstimateGroupNetwork/index.html

Davis, M. H. (1980). A multidimensional approach to individual differences in empathy. JSAS Catalog of Selected Documents in Psychology, 10, 85.

Efroymson, M. A. (1960). Multiple regression analysis. In A. Ralston \& H. S. Wilf (Eds.), Mathematical methods for digital computers (pp. 191-203). New York: Wiley.

Epskamp, S., Cramer, A. O., Waldorp, L. J., Schmittmann, V. D., \& Borsboom, D. (2012). qgraph: Network visualizations of relationships in psychometric data. Journal of Statistical Software, 48, 1-18. http://dx .doi.org/10.18637/jss.v048.i04

Epskamp, S., \& Fried, E. I. (2018). A tutorial on regularized partial correlation networks. Psychological Methods, 23 (4), 617-634. doi:10.1037/met0000167

Forbes, M. K., Wright, A. G. C., Markon, K. E., \& Krueger, R. F. (2017). Evidence that psychopathology symptom networks have limited replicability. Journal of Abnormal Psychology, 126 (7), 969-988. doi: 10.1037/abn0000276 
Forbes, M. K., Wright, A. G. C., Markon, K. E., \& Krueger, R. F. (2019). Quantifying the reliability and replicability of psychopathology network characteristics. Multivariate Behavioral Research. doi: 10.1080/00273171.2019.1616526

Fried, E. I., \& Nesse, R. M. (2014). The impact of individual depressive symptoms on impairment of psychosocial functioning. PLoS One, 9 (2), e90311. doi:10.1371/journal.pone.0090311

Fried, E. I., van Borkulo, C. D., Cramer, A. O. J., Boschloo, L., Schoevers, R. A., \& Borsboom, D. (2017). Mental disorders as networks of problems: a review of recent insights. Social Psychiatry and Psychiatric Epidemiology, 52, 1-10. doi:10.1007/s00127-016-1319-z

Fried, E. I., Eidhof, M. B., Palic, S., Costantini, G., Huisman-van Dijk, H. M., Bockting, C. L. H., Engelhard. I., Armour, C., Nielsen, A. B. S., Karstoft, K.-I. (2018). Replicability and generalizability of posttraumatic stress disorder (PTSD) networks: a cross-cultural multisite study of PTSD symptoms in four trauma patient samples. Clinical Psychological Science, 6 (3), 335-351. doi: 10.1177/2167702617745092

Furnival, G. M., \& Wilson, R. W. (1974). Regression by leaps and bounds. Technometrics, 16 (3), 499-512.

Garside, M. J. (1971). Some computational procedures for the best subset problem. Applied Statistics, 20 (1), 8-15.

Goodnight, J. H. (1979). A tutorial on the SWEEP operator. The American Statistician, 33 (3), 149-158.

Grömping, U. (2006). Relative importance for linear regression in R: The package relaimpo. Journal of Statistical Software, 17, 1-27.

Grömping, U. (2015). Variable importance in regression models. WIREs Computational Statistics, 7, 137-152. doi: 10.1002/wics.1346

Hebbali, A. (2020). Package olsrr: Tools for building OLS regression models. Retrieved from: https://cran.r-project.org/web/packages/olsrr/olsrr.pdf

Heeren, A., \& McNally, R. J. (2016). An integrative network approach to social anxiety disorder: The complex dynamic interplay among attentional bias for threat, attentional control, and symptoms. Journal of Anxiety Disorders, 42, 95-104. doi: 10.1016/ j.janxdis.2016.06.009

Hoorelbeke, K., Marchetti, I., De Schryver, M., \& Koster, E. H. W. (2016). The interplay between cognitive risk and resilience factors in remitted depression: A network analysis. Journal of Affective Disorders, 195, 96-104. doi: 10.1016/j.jad.2016.02.001

LaMotte, L. R., \& Hocking, R. R. (1970). Computational efficiency in the selection of regression variables. Technometrics, 12 (1), 83-93.

Lindeman, R. H., Mirenda, P. F., \& Gold, R. Z. (1980). Introduction to Bivariate and Multivariate Analysis. Glenview, IL: Scott, Foresman.

Loeffelman, J. E., Steinley, D., Boness, C. L., Trull, T. J., Wood, P. K., Brusco, M. J., \& Sher, K. J. (2020). Combinatorial optimization of clustering decisions: An approach to refine psychiatric diagnoses. Multivariate Behavioral Research, doi:

10.1080/00273171.2020.1717921 
Lumley, T., \& Miller, A. (2020). Package leaps: Regression subset selection. Retrieved from: https://cran.r-project.org/web/packages/leaps/leaps.pdf

Mallows, C. L. (1973). Some comments on $C_{p}$. Technometrics, 15 (4), 661-675. doi:10.1080/00401706.1973.10489103

MATLAB. (2020). version 9.8.0 (R2020a). Natick, Massachusetts: The MathWorks Inc.

McNally, R. J. (2016). Can network analysis transform psychopathology? Behaviour Research and Therapy, 86, 95-104. doi: 10.1016/j.brat.2016.06.006

McNally, R. J., Robinaugh, D. J., Wu, G. W. Y., Wang, L., Deserno, M. K., \& Borsboom, D. (2015). Mental disorders as causal systems: A network approach to posttraumatic stress disorder. Clinical Psychological Science, 3 (6), 836-849. doi:10.1177/2167702614553230

Miller, A. J. (2002), Subset Selection in Regression (2nd ed.). London: Chapman and Hall.

Nimon, K. F., \& Oswald, F. L. (2013). Understanding the results of multiple linear regression: Beyond standardized regression coefficients. Organizational Research Methods, 16 (4), 650-674. 10.1177/1094428113493929

Robinaugh, D. J., LeBlanc, N. J. Vuletich, H. A., \& McNally, R. J. (2014). Network analysis of persistent complex bereavement disorder in conjugally bereaved adults. Journal of Abnormal Psychology, 123 (3), 510-522. doi: 10.1037/abn0000002

Schwartz, G. (1978). Estimating the dimension of a model. Annals of Statistics, 6 (2), 461-464.

Scutari, M. (2010). Learning Bayesian networks with the bnlearn R package. Journal of Statistical Software, 35, 1-22. Retrieved from http:// www.jstatsoft.org/v35/i03/

Smith, D. M. (1991). Algorithm AS 268, all possible subset regressions using the QR decomposition. Applied Statistics, 40 (3), 502-513. doi:10.2307/2347539

van Borkulo, C. D., Borsboom, D., Epskamp, S., Blanken, T. F., Boschloo, L., Schoevers, R. A., Waldorp, L. J. (2014). A new method for constructing networks from binary data. Scientific Reports, 4, 5918; DOI:10.1038/srep05918.

van den Berg, J. W., Smid, W., Kossakowski, J. J., van Beek, D., Borsboom, D., Janssen, E., \& Gijs, L. (2020). The application of network analysis to dynamic risk factors in adult male sex offenders. Clinical Psychological Science, 8 (3), 539-554. doi:10.1177/2167702620901720

Williams, D. R., \& Rast, P. (2020). Back to the basics: Rethinking partial correlation network methodology. British Journal of Mathematical and Statistical Psychology, 73 (2), 187-212. doi: 10.1111/bmsp.1217

Williams, D. R., Rhemtulla, M., Wysocki, A. C., \& Rast, P. (2019). On nonregularized estimation of psychological networks. Multivariate Behavioral Research, 54 (5), 719-750. doi: 10.1080/00273171.2019.1575716 